\title{
PERSPECTIVE
}

\section{Using empirically-derived dimensional phenotypes to accelerate clinical neuroscience: the Hierarchical Taxonomy of Psychopathology (HiTOP) framework}

\author{
Robert D. Latzman (D) ${ }^{1}$, Colin G. DeYoung ${ }^{2}$ and The HiTOP Neurobiological Foundations Workgroup
}

Neuropsychopharmacology (2020) 45:1083-1085; https://doi.org/10.1038/s41386-020-0639-6

Clinical neuroscience seeks to understand the neurobiological mechanisms of mental illness and translate that knowledge into effective, biologically-informed interventions, including pharmacological approaches. The past two decades have witnessed the development of powerful new tools for quantifying variation in the human brain. Nonetheless, although substantial resources and efforts have been invested in identifying reliable neurobiological indicators of mental illness, or "biomarkers", only modest progress has been made. A growing consensus attributes this lack of progress largely to the shortcomings of current categorical diagnostic systems, rather than intrinsic limitations of biological approaches [1].

Categorical diagnoses are suboptimal targets for research on the foundations of psychopathology, and subsequently on the development of biologically-informed interventions, due to their extensive comorbidity, within-disorder heterogeneity, and poor reliability [2]. Within-disorder heterogeneity is so extreme that, for many disorders, two patients may receive the same diagnosis despite sharing few or no overlapping symptoms. Further, no common mental disorder has ever been shown to be categorical rather than dimensional, and the cutoffs used to define diagnoses lack empirical justification, reduce statistical power, and reduce reliability and validity relative to the use of dimensional scores [2].

The U.S. National Institutes of Health (NIH) have responded to this crisis with the NIMH Research Domain Criteria, the NIAAA Addictions Neuroclinical Assessment, and the NIDA Phenotyping Assessments Battery-diagnostically agnostic frameworks formulated by groups of experts around transdiagnostic, biobehavioral systems. Although they place valuable emphasis on neural systems, such frameworks are currently limited in their definition of relevant clinical presentations of mental illness; thus, the relation of these frameworks to patients' suffering, dysfunction in daily life, and potential treatment remains unclear. A complementary framework is needed to link data on biobehavioral systems to the patterns of signs and symptoms that lead people to seek treatment.

An empirically derived, dimensional model of psychopathology can provide just such a framework, working together with the $\mathrm{NIH}$ frameworks synergistically to boost their effectiveness. Integrating decades of nosological and psychometric research, the Hierarchical Taxonomy of Psychopathology (HiTOP; https://medicine. stonybrookmedicine.edu/HITOP) consortium has developed a hierarchical, dimensional model with the potential to encompass the full range of psychopathology (Fig. 1) [2]. At the top levels of the HiTOP hierarchy are broad dimensional spectra and superspectra, including an overarching general psychopathology dimension that reflects broad liability to diverse forms of psychopathology situated lower in the hierarchy. The internalizing spectrum, for example, encompasses more specific subfactors including fear and distress, which, in turn, encompass even narrower clinical phenotypes (e.g., social anxiety, insomnia). Researchers are thus able to test relations with neurobiological mechanisms at multiple levels of the hierarchy in a single study (Fig. 1). Different biological parameters are likely to confer risk at different levels, such that parameters linked to higher levels influence a wider range of related behaviors than those linked to lower levels. This kind of differential pattern has now been observed in a number of MRI studies e.g., refs. [3, 4]. To describe one example, a study of cerebral blood flow in over 1000 youth found that the general factor of psychopathology was associated with elevated perfusion in the anterior cingulate and several other brain regions, whereas subdimensions of fear and psychosis were uniquely related to lower perfusion in the insula and fusiform gyrus, respectively [4].

The HiTOP model provides clinical phenotypes considerably more informative for neuroscience research generally, and for research on potential pharmacological treatments specifically, than current categorical diagnostic systems [2]. HiTOP addresses diagnostic comorbidity by directly modeling which aspects of psychopathology systematically co-occur. It addresses heterogeneity through its hierarchical structure, with broad dimensions at higher levels subdivided into narrower subdimensions at lower levels. Symptoms are organized empirically, such that closely correlated symptoms are assigned to the same dimension and unrelated symptoms to different dimensions. These features, along with the use of dimensions in place of categories, foster increased reliability and precision of measurement. Not surprisingly, therefore, neuroscience research using dimensional constructs consistent with HiTOP is beginning to provide results that appear stronger and more replicable than results from research on categorical diagnoses. For example, meta-analysis identified disruption in fronto-amygdala connectivity as a transdiagnostic neural signature of internalizing psychopathology [5], and a transdiagnostic study of psychotic disorders found that symptom dimensions predict biological differences twice as well as diagnoses do [6]. Much of this research uses dimensional

\footnotetext{
${ }^{1}$ Georgia State University, Atlanta, GA, USA and ${ }^{2}$ University of Minnesota, Minneapolis, MN, USA

Correspondence: Robert D. Latzman (rlatzman@gsu.edu) or Colin G. DeYoung (cdeyoung@umn.edu)

These authors contributed equally: Robert D. Latzman, Colin G. DeYoung
}

Received: 12 October 2019 Revised: 3 February 2020 Accepted: 5 February 2020

Published online: 28 February 2020 


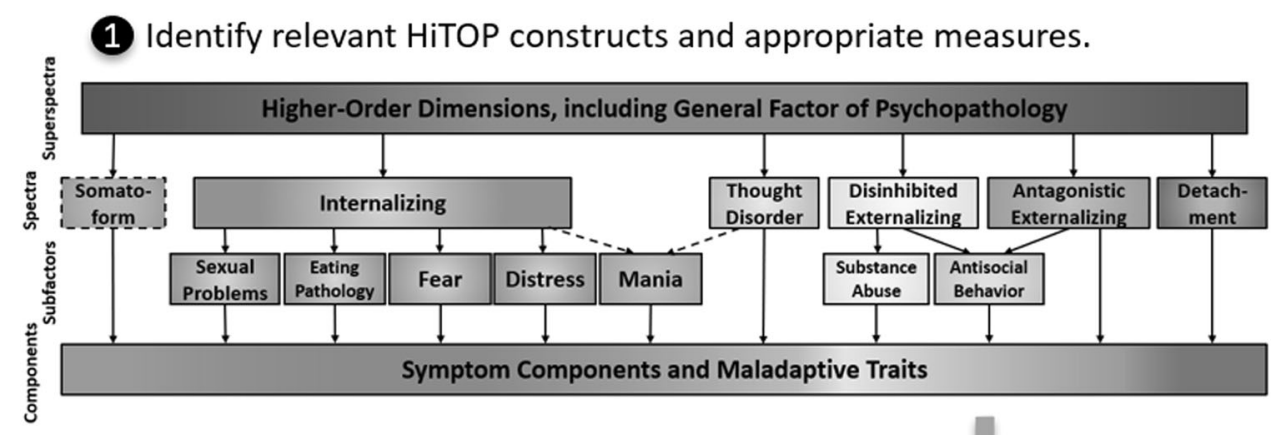

(2) Assess in representative population, potentially oversampling from range of maximal clinical relevance.

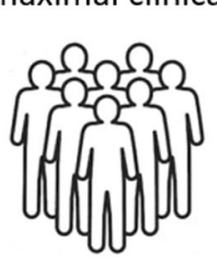

Dimension of Interest
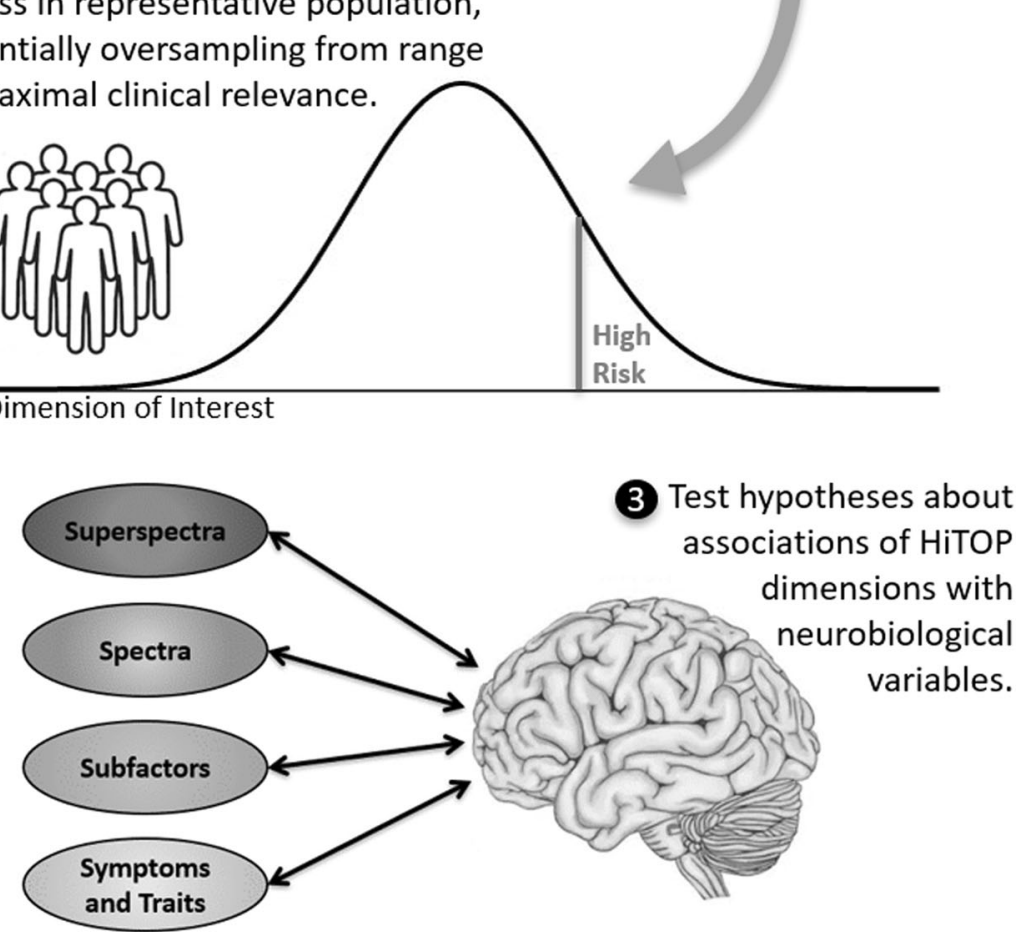

Fig. 1 Using the Hierarchical Taxonomy of Psychopathology (HiTOP) in clinical neuroscience. Recent efforts by an international consortium of researchers have led to HiTOP, a consensual dimensional system (https://medicine.stonybrookmedicine.edu/HITOP/). Step 1 depicts a simplified schematic of the HiTOP working model, from which clinical phenotypes should be selected for study. (HiTOP is a work in progress and will be updated on the basis of new data. Dashed lines indicate provisional elements requiring more study.) Step 2 depicts a sampling design appropriate for HiTOP-based research, which involves sampling from unselected patient populations or the general population, rather than a case-control design, although researchers may wish to oversample participants manifesting or at high risk for the problems of interest. Step 3 depicts testing associations between HiTOP phenotypes and neurobiological variables, ideally examining constructs at multiple levels of the hierarchy, examining constructs from multiple spectra to assess discriminant validity, and using latent variable modeling.

measures that predate HiTOP, but HiTOP provides the integrative framework that describes their relation to other forms of psychopathology. Although the majority of this research to date has been carried out without a pharmacological component, clinical pharmacological research stands to benefit similarly from HiTOP.

In the absence of a guiding nosological model, progress in clinical neuroscience will be difficult. Prior to the development of HiTOP, there was no model capable of organizing the full range of dimensional, transdiagnostic phenotypes based on the covariation of clinical signs, symptoms, and diagnoses. HiTOP provides a framework for linking clinical phenotypes with measures of neurobiological systems, allowing integration of the various constructs targeted by $\mathrm{NIH}$ initiatives. We recommend, therefore, that clinical neuroscientists familiarize themselves with HiTOP and plan studies that incorporate measures of constructs appearing within it, preferably assessing multiple HiTOP spectra in order to investigate discriminant validity. When possible, they should also use measures that capture lower as well as higher levels of the hierarchy, potentially using self-report as well as interview-based measures. Clinical neuroscience studies should avoid case-control designs based on categorical diagnoses (in which the differences between cases and controls on the dimensions of interest are confounded by many extraneous factors) and focus instead on sampling from unselected patient populations or the general population, potentially oversampling participants in the range of high risk on the dimensions of interest. In addition to facilitating study design, HiTOP will also aid development of hypotheses and interpretation of results because it facilitates synthesis of existing research in clinical neuroscience by showing which clinical constructs cohere in empirically validated dimensions, thereby often allowing integrative inferences across studies that used different measures and constructs. In sum, HiTOP is poised to accelerate progress in clinical neuroscience, providing a bridge between basic neuroscience research and clinically-relevant dimensions of psychopathology. 
FUNDING AND DISCLOSURE

The authors declare no competing interests.

\section{AUTHOR CONTRIBUTIONS}

The HiTOP Neurobiological Foundations Workgroup (MH Afzali, TA Allen, RR Althoff, CG DeYoung, AR Docherty, M Dretsch, NR Eaton, VM Goghari, RG Grazioplene, MN Hallquist, JD Haltigan, AS Heller, AJ Holmes, R Kotov, RF Krueger, RD Latzman, EA Martin, G Michelini, CJ Patrick, AC Ruocco, AJ Shackman, JL Tackett, MT Treadway, NC Venables, ID Waldman, and DH Zald, listed in alphabetical order) collectively determined the overall structure of the paper. RDL and CGD drafted the paper and share first-authorship. All authors reviewed and approved the final version of the paper.

\section{ADDITIONAL INFORMATION}

Publisher's note Springer Nature remains neutral with regard to jurisdictional claims in published maps and institutional affiliations.

\section{REFERENCES}

1. Gordon JA, Redish AD. On the cusp. Current challenges and promises in psychiatry. In: Redish AD, Gordon JA, editors. Computational psychiatry: new perspectives on mental illness. Cambridge, MA: MIT Press; 2016. p. 3-14.

2. Krueger RF, Kotov R, Watson D, Forbes MK, Eaton NR, Ruggero $\mathrm{CJ}$, et al. Progress in achieving empirical classification of psychopathology. World Psychiatry. 2018;17:282-93.

3. Castellanos-Ryan N, Struve M, Whelan R, Banaschewski T, Barker GJ, Bokde ALW, et al. Neural and cognitive correlates of the common and specific variance across externalizing problems in young adolescence. Am J Psychiatry. 2014;171:1310-9.

4. Kaczkurkin AN, Moore TM, Calkins ME, Ciri R, Detre JA, Elliott MA, et al. Common and dissociable regional cerebral blood flow differences associate with dimensions of psychopathology across categorical diagnoses. Mol Psychiatry. 2018;23:1981-9.

5. Marusak HA, Thomason ME, Peters C, Zundel C, Elrahal F, Rabinak CA. You say 'prefrontal cortex' and I say 'anterior cingulate': meta-analysis of spatial overlap in amygdala-to-prefrontal connectivity and internalizing symptomology. Transl Psychiatry. 2016;6:e944.

6. Reininghaus U, Böhnke JR, Chavez-Baldini U, Gibbons R, Ivleva E, Clements BA, et al. Trandiagnostic dimensions of psychosis in the Bipolar-Schizophrenia Network on Intermediate Phenotypes (B-SNIP). World Psychiatry. 2019;18:67-76.

\section{THE HITOP NEUROBIOLOGICAL FOUNDATIONS WORKGROUP}

M. H. Afzali ${ }^{3}$, T. A. Allen ${ }^{4}$, R. R. Althoff ${ }^{5}$, C. G. DeYoung ${ }^{6}$, A. R. Docherty ${ }^{7}$, M. Dretsch ${ }^{8}$, N. R. Eaton ${ }^{9}$, V. M. Goghari ${ }^{10}$, R. G. Grazioplene ${ }^{11}$, M. N. Hallquist ${ }^{4}$, J. D. Haltigan ${ }^{10}$, A. S. Heller ${ }^{12}$, A. J. Holmes ${ }^{11}$, R. Kotov ${ }^{9}$, R. F. Krueger ${ }^{6}$, R. D. Latzman ${ }^{13}$, E. A. Martin ${ }^{14}$, G. Michelini ${ }^{9}$, C. J. Patrick ${ }^{15}$, A. C. Ruocco ${ }^{10}$, A. J. Shackman ${ }^{16}$, J. L. Tackett $^{17}$, M. T. Treadway ${ }^{18}$, N. C. Venables ${ }^{6}$, I. D. Waldman ${ }^{18}$ and D. H. Zald $^{19}$

${ }^{3}$ University of Montreal, Montreal, Canada. ${ }^{4}$ Pennsylvania State University, Pennsylvania, USA. ${ }^{5}$ University of Vermont, Burlington, USA. ${ }^{6}$ University of Minnesota, Minneapolis, MN, USA. 'University of Utah, Salt Lake City, USA. ${ }^{8}$ US Army Medical Research Directorate-West, Washington, USA. ${ }^{9}$ Stony Brook University, New York, USA. ${ }^{10}$ University of Toronto, Ontario, Canada. ${ }^{11}$ Yale University, New Haven, USA. ${ }^{12}$ University of Miami, Coral Gables, USA. ${ }^{13}$ Georgia State University, Atlanta, GA, USA. ${ }^{14}$ University of California, Irvine, USA. ${ }^{15}$ Florida State University, Tallahassee, USA. ${ }^{16}$ University of Maryland, College Park, USA. ${ }^{17}$ Northwestern University, Evanston, USA. ${ }^{18}$ Emory University, Oxford, USA. ${ }^{19}$ Vanderbilt University, Nashville, USA. 